Portland State University

PDXScholar

6-9-1993

\title{
Waveform Estimation with Jitter Noise by Pseudo Symmetrical Probability Density Function
}

Wei-Da Hao

Portland State University

Follow this and additional works at: https://pdxscholar.library.pdx.edu/open_access_etds

Part of the Electrical and Electronics Commons

Let us know how access to this document benefits you.

\section{Recommended Citation}

Hao, Wei-Da, "Waveform Estimation with Jitter Noise by Pseudo Symmetrical Probability Density Function" (1993). Dissertations and Theses. Paper 4587.

https://doi.org/10.15760/etd.6471

This Thesis is brought to you for free and open access. It has been accepted for inclusion in Dissertations and Theses by an authorized administrator of PDXScholar. Please contact us if we can make this document more accessible: pdxscholar@pdx.edu. 
AN ABSTRACT OF THE THESIS OF Wei-Da Hao for the Master of Science in Electrical Engineering presented June 9, 1993.

Title: Waveform Estimation with Jitter Noise by Pseudo Symmetrical Probability Density Function

APPROVED BY THE MEMBERS OF THE THESIS COMMITTEE:
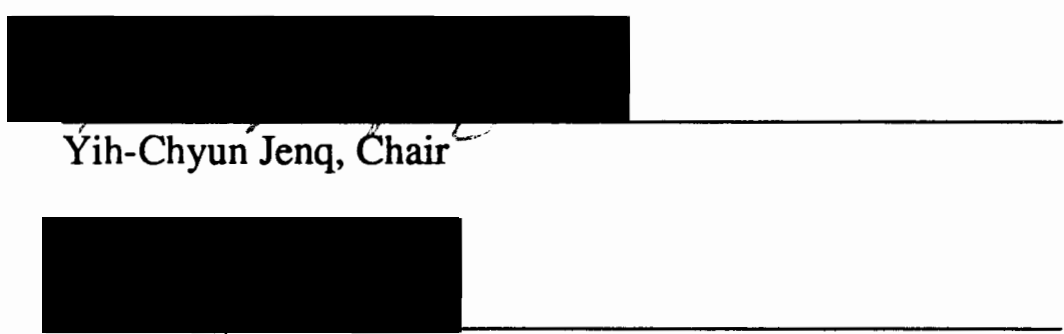

Richard P. E. Tymerski

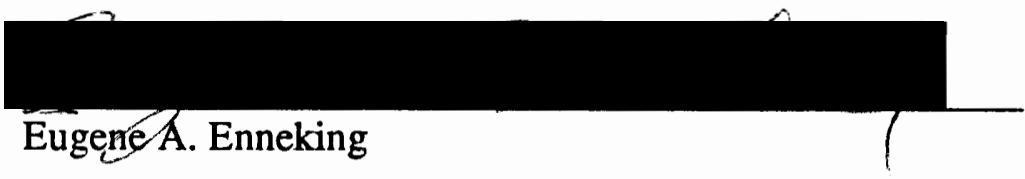

A new method for solving jitter noise in estimating high frequency waveform is proposed. It reduces the bias of the estimation in those points where all the other methods fail to achieve.

It provides preliminary models for estimating percentiles in Normal, Exponential probability density function. Based on the model for Normal probability density function, a model for any probability density function is derived. The resulting percentiles, in turn, are used as estimates for the amplitude of the waveform. Simulation results show us with satisfactory accuracy. 
WA VEFORM ESTIMATION WITH JITTER NOISE

BY PSEUDO SYMMETRICAL PROBABILITY DENSITY FUNCTION

by

WEI-DA HAO

A thesis submitted in partial fulfillment of the requirement for the degree of

MASTER OF SCIENCE

in

ELECTRICAL ENGINEERING

Portland State University

1993 
TO THE OFFICE OF GRADUATE STUDIES:

The members of the Committee approve the thesis of Wei-Da Hao presented June 9, 1993.

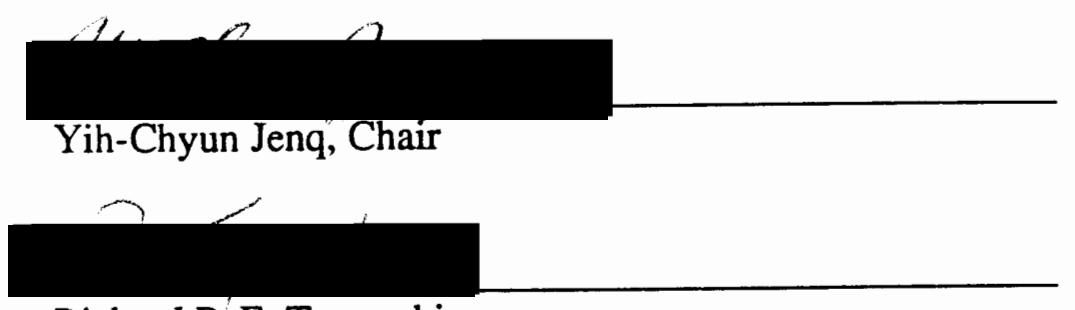

Richard P. E. Tymerski

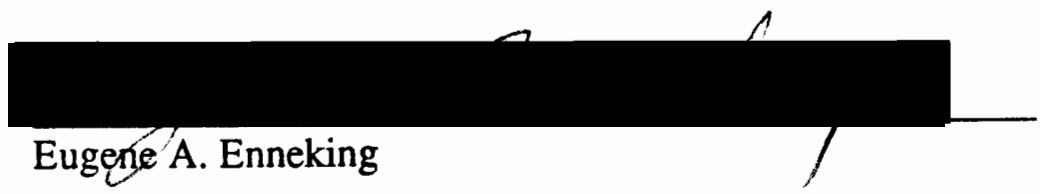

APPROVED:

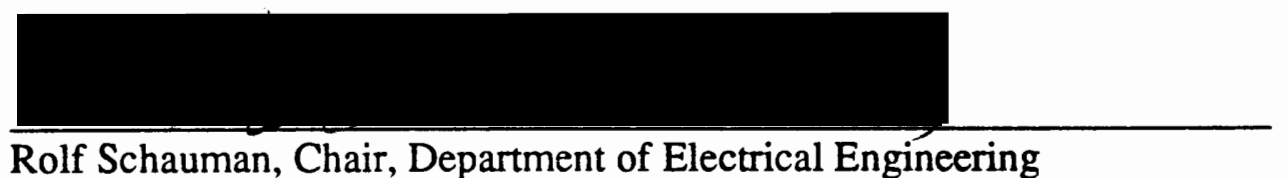

Rolf Schauman, Chair, Department of Electrical Engineering

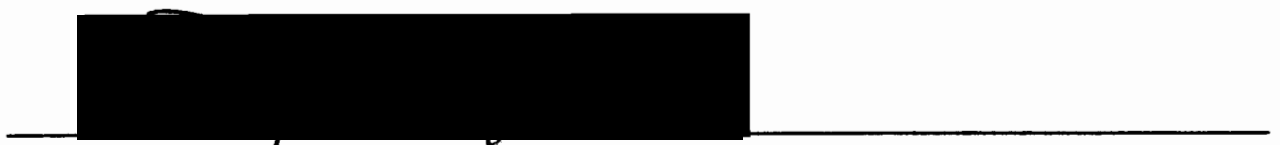

Roy W. Koch, Wice Provost for Graduate Studies and Research 


\section{ACKNOWLEDGEMENTS}

I am grateful to many, many authors who have enriched the field of Instrumentation and Measurement with their writings, and on whose works I have drawn freely. My compliment to all of those who have shared their ideas and comments regarding Jitter Noise and Markov Chain, especially my advisor Dr. Y. C. Jenq. His insight, patient discussion and continuing encouragement make the achievement in this thesis possible. Thanks also to M. T. Souders at NIST. Without his communicating letter, the initial phases of our studies would have been very difficult. I further acknowledge and thank to the secretarial staff of the Electrical Engineering Department, who have kindly provide all-around administrative care.

My thanks to Mr. David Tupper are commensurate with those to the people above. He let me be a member of his family, sharing loving and caring. With his family in my mind I feel Portland is my home.

Last but not the least, I am indebted to my parents. With their support, I can continue graduate education in the United States. 
TABLE OF CONTENTS

PAGE

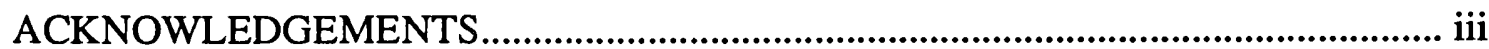

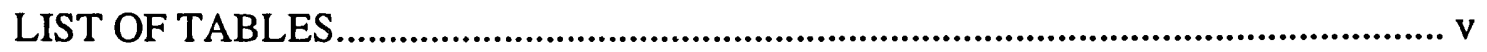

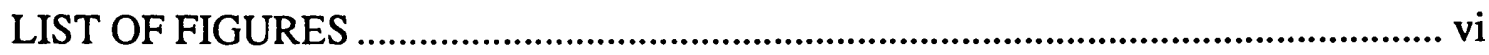

CHAPTER

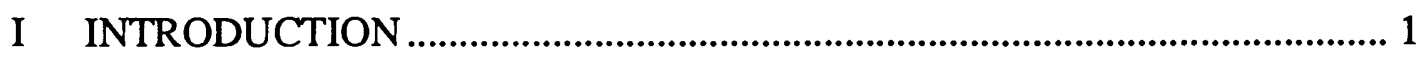

Markov Estimator( Basic Up and Down Method ).......................................... 4

Markov Estimator with Compensation................................................................ 4

Approximation, Up and Down in Generalized Form,

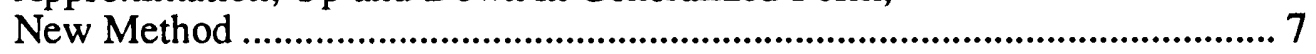

II STOCHASTIC APPROXIMATION METHOD.......................................... 9

III STOCHASTIC UP AND DOWN METHOD

IN GENERALIZED FORM ................................................................ 13

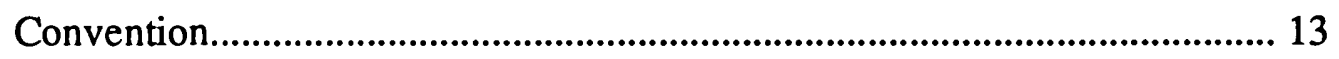

Some Properties of Stochastic Up and Down Method ....................................... 14

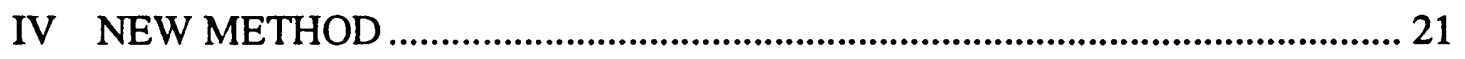

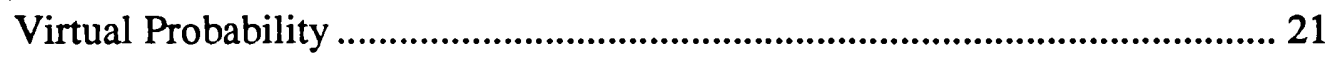

New Method

- Virtually Generate Dynamically Symmetric P.D.F. .............................. 22

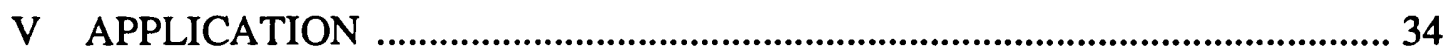

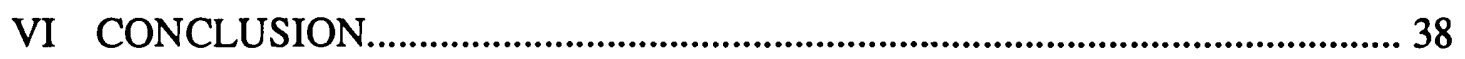

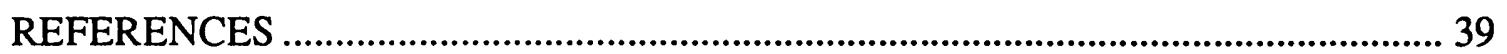




\section{LIST OF TABLES}

I Percentile Estimation with Different Methods on

Gaussian Distribution Repetitive Times $10^{7}$

11

II Percentile Estimation with Different Methods on

Exponential Distribution Repetitive Times $10^{7}$

12

III Distribution of $\mathrm{N}(0,1)$ Modified to $\mathrm{Be}$

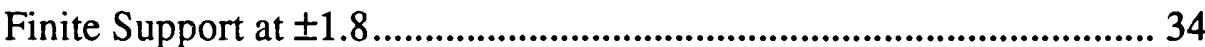

IV Bias of Waveform Estimation for

$$
y=\sin (0.1 \times t)-0.1 \times \sin (0.3 \times t)
$$




\section{LIST OF FIGURES}

FIGURE

PAGE

1. Illustration of the Bias Associated with Using the Mean

to Estimate the Value of a Waveform Sampled at Time $t$, with Timing Jitter Having a PDF of $\mathrm{P}(\Delta \mathrm{t})$........................................ 2

2. Illustration of Source of Jitter Noise Error............................................... 5

3. Bias of Percentile Estimation for Exponential Distribution $\mathrm{N}=10^{7}, \delta=10^{-1}$ 30

4. Bias of Percentile Estimation for Exponential Distribution $\mathrm{N}=10^{7}, \delta=10^{-2}$ 31

5. Bias of Percentile Estimation for Normal Distribution $\mathrm{N}=10^{7}, \delta=10^{-1}$ 32

6. Bias of Percentile Estimation for Normal Distribution $\mathrm{N}=10^{7}, \delta=10^{-2}$ 33

7. Sample $\sin (0.1 \times t)-0.1 \times \sin (0.3 \times t)$

with Jitter Distribution N(0,1)

Modified to be Finite Support at \pm 1.8

8. Bias of Waveform Estimation with Jitter Noise $\mathrm{N}=10^{7}$ 


\section{CHAPTER I}

\section{INTRODUCTION}

Jitter noise exists everywhere, especially in sampling high frequency waveforms. It is induced by the failure of the instrument itself to keep pace with the speed of the signal. Timing jitter generally causes systematic errors in the amplitude estimates of sampled waveforms. This is true for both real and equivalent-time sampling processes. An example is the tendency to delay or overestimate on the response of the trigger signal. If the frequency of the signal is low, e.g. less than $1 \mathrm{MHz}$, then the effect of the jitter noise is not explicit.

For repetitive waveform we can obtain the real waveform by analyzing a group of data from a jitter distribution. Usually, the jitter distribution is normal. The deviation from the mean represents the delaying or overestimating by the probing instrument. Consider the case of equivalent time sampling : Multiple samples are often taken at each nominal sample time and, to reduce the effects of noise, the mean of each sample set is taken as the estimated value at the corresponding sample time. If additive noise with zero mean exists in the sample, then the resulting estimates are unbiased. However, if the noise is the result of timing jitter, then the mean generally gives rise to systematic errors in the estimates. In Figure 1 the bias of using the mean is depicted. If , on the other hand, the median, rather than the mean, is used to compute the estimates, then bias occurs only for signals which are non-monotonic. 


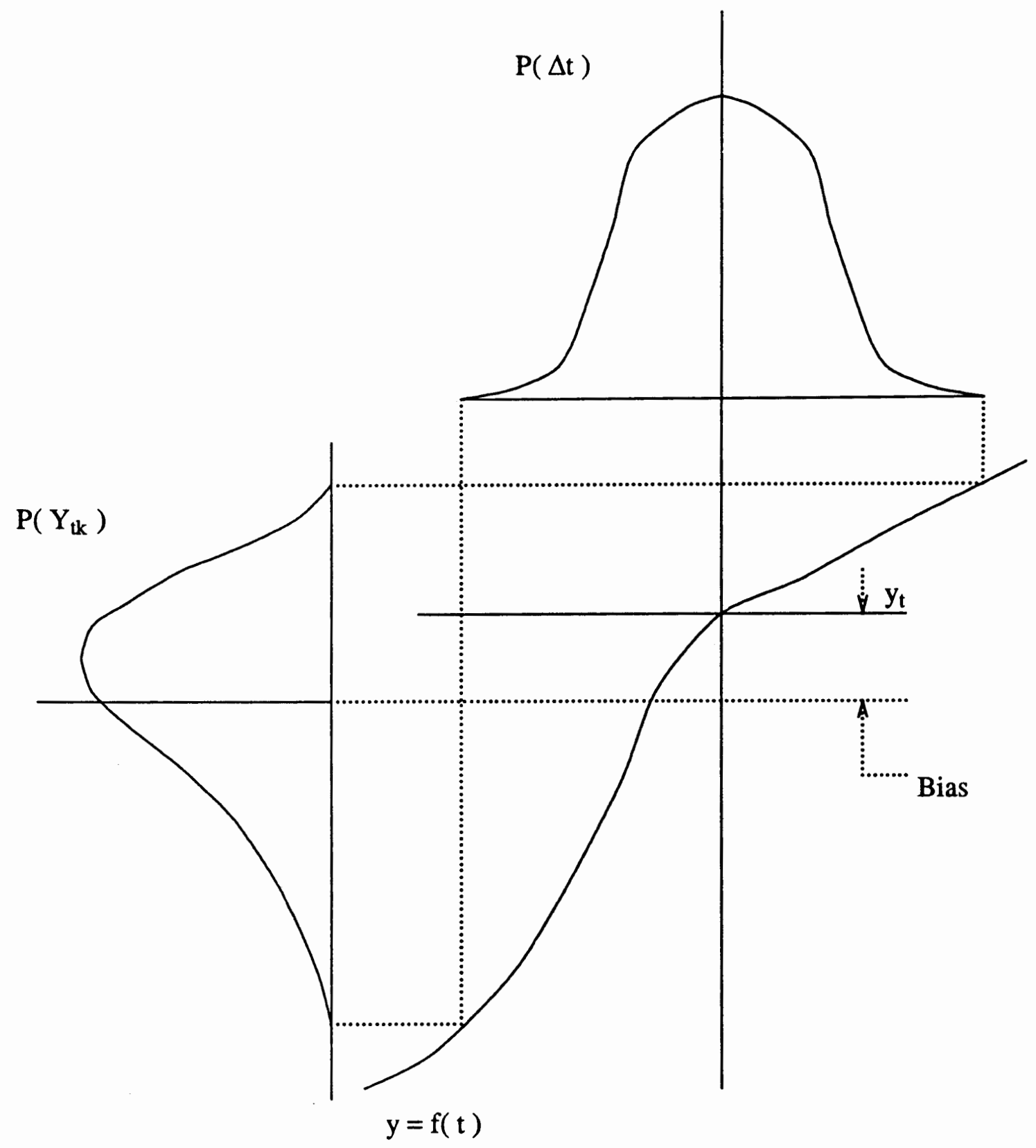

Figure 1. Illustration of the bias associated with using the mean to estimate the value of a waveform sampled at time $t$, with timing jitter having a PDF of $P(\Delta t)$.

A deconvolution method by Gans [1] is the first appearance in the literature aimed toward solving jitter noise. However, a potential problem exists which reduces its applicability. 
Recently, people in NIST applied an Up and Down method to deal with this problem. The output of this method is an estimator named "Markov estimator". In 1.1 we discuss this method. The literature is extensive on properties of "Markov estimator", but their presentation is on the advanced level [2]. They point out the concentration of the "Markov estimator". This method provides an alternative way to analyze a waveform. However, this method causes a lot of bias, when the waveform is not monotonic. There is explicit bias near the peak area. To acquire more reliable results, Tong and Souders proposed an improved method using "Markov estimator with compensation" that can compensate the bias when estimating the waveform near the peak. In 1.2 we discuss this method in detail. In their method, if the time waveform peak occurs and the jitter distribution are known before hand by some preprocessing, the bias is reduced to a satisfactory amount. Although this is a good way to resolve the jitter noise without using the deconvolution up to now, it is not well-developed in the theory : this method has uncertainty regarding the value $t_{d}$. We can discern this fact through the equations listed in 1.2.

In the rest of this chapter we give an introduction to the Markov estimator, the Markov estimator with compensation and the alternative methods. The reason for proposing alternative methods is stated in 1.3.

The subsequent chapters will be devoted to detailed discussion about "Stochastic Up and Down", "Up and Down in Generalized Form" and "New Method". After analyzing simulation results, we recommend "Up and Down in Generalized Form" and "New Method" in solving jitter noise. When considering the accuracy of some specified estimated points, the "New Method" is the most appropriate one. 


\section{MARKOV ESTIMATOR ( BASIC UP AND DOWN METHOD )}

The Markov estimator is based on a Markov process which operates on the statistical sample set. The Markov process works as follows. Let $\mathrm{y}_{\mathrm{t}}(\mathrm{k}), \mathrm{k}=0,1,2, \cdots$ be a sample of the input waveform $Y(t)$ all taken at the nominal sample time, $t$, computing in turn with the reference value, $y_{t}^{\prime}(k)$. This reference value is incremented or decremented by a small fixed amount, $\delta$, after each sample, depending on the state of the previous comparison. Under these conditions, $y_{t}^{\prime}(k), k=0,1,2, \cdots$ follows a random walk described by a Markov chain, defined as

$$
\begin{aligned}
& y_{t}^{\prime}(k+1)=y_{t}^{\prime}(k)+\delta \text { for } y_{t}^{\prime}(k) \leq y_{t}(k) \\
& y_{t}^{\prime}(k+1)=y_{t}^{\prime}(k)-\delta \text { for } y_{t}^{\prime}(k)>y_{t}(k)
\end{aligned}
$$

The Markov estimator, $Y^{\prime}(t)$, is the mean value of the Markov chain, $y_{t}^{\prime}(k), k=0,1,2, \cdots$ :

$$
Y^{\prime}(t)=\frac{1}{n+1} \sum_{k=0}^{n} y_{t}^{\prime}(k)
$$

$\mathrm{Y}^{\prime}(\mathrm{t})$ asymptotically converges to the population median of $\mathrm{y}_{\mathrm{t}}(\mathrm{k})$, as $\mathrm{n} \rightarrow \infty$ and then $\delta \rightarrow 0[3]$.

\section{MARKOV ESTIMATOR WITH COMPENSATION [4]}

The source of the errors produced by the Markov estimator in the presence of timing jitter is illustrated in Figure 2.

A non-monotonic region of the input signal, $Y(t)$, is shown, sampled at time $t$. The sampling is subject to timing jitter having a probability density function ( PDF ) 


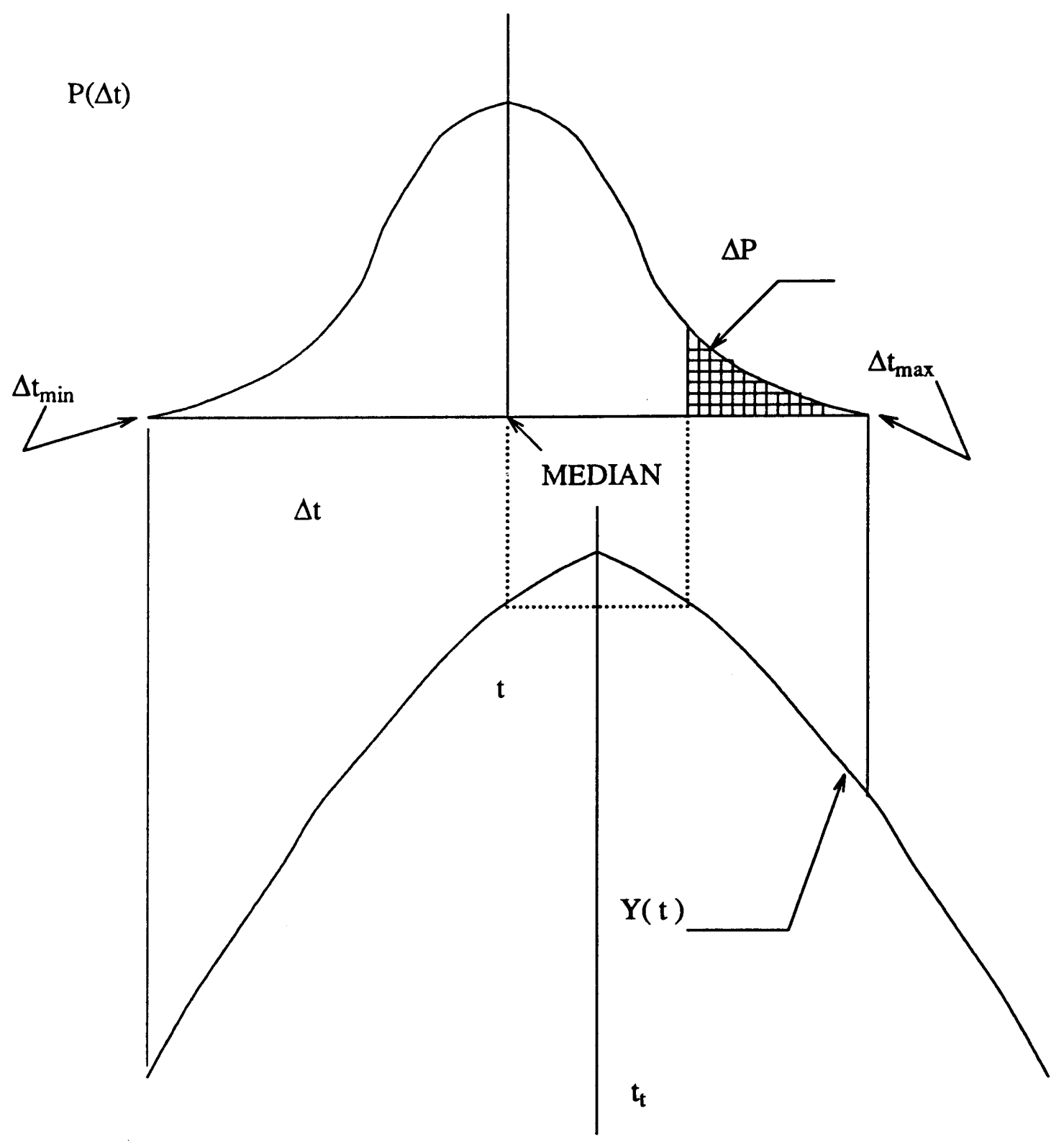

Figure 2. Illustration of Source of Jitter Noise Error.

given by $p(\Delta t)$ with a median of 0 . For the following analysis, it will be assumed that $Y(t)$ is symmetrical about time $t_{p}$ ( the stationary point where $d Y(t) / d t=0$ ) over the region defined by $t_{p} \pm \Delta t_{p k} / 2$, where $\Delta t_{p k}$ is the larger of $\left|\Delta t_{\max }\right|$ or $\left|\Delta t_{\min }\right|$, and that all stationary points are separated by at least $\Delta \mathrm{t}_{\mathrm{pk}}$. It can be seen from the figure 
that, if point $t$ is at least $\frac{\Delta t_{p k}}{2}$ away from $t_{p}$, then half of the samples, $Y(t+\Delta t)$, will be greater than $Y(t)$, and half will be less. Therefore, the Markov estimator, $Y^{\prime}(t)$, defined as the median of samples $Y(t+\Delta t)$, will converge to $Y(t)$ in the limit, i.e., it will be asymptotically unbiased. However, when the nominal sample time, $t$, is within $\frac{\Delta t_{\max }}{2}$ on the left side, or $\frac{\Delta t_{\min }}{2}$ on the right site of time $t_{p}$, some of the samples taken on one side of the probability distribution ( designated by $\Delta P$ in the figure ) will be greater than $Y(t)$ and some will be less. Thus, the median, $Y^{\prime}(t)$, of the samples, i.e., the Markov estimator, will not be an unbiased estimate of $Y(t)$. The fraction of the samples, $y_{t}(k)$, that have changed to be less than median is given by

$$
\Delta P=\int_{2\left(t_{p}-t\right)}^{\Delta t_{\max }} p(\Delta t) d(\Delta t) \quad\left(t_{1}<t<t_{p}\right)
$$

for $t<t_{p}$, where $t_{1}$ is $t_{p}-\Delta t_{p} k / 2$ and by

$$
\Delta P=\int_{\Delta t_{\min }}^{2\left(t_{p}-t\right)} p(\Delta t) d(\Delta t) \quad\left(t_{p}<t<t_{2}\right)
$$

for $t>t_{p}$, where $t_{2}$ is $t_{p}+\Delta t_{p k} / 2$

The Markov estimator, $Y^{\prime}(t)$, is the same value as the level $Y\left(t+t_{d}\right)$, where the time shift $t_{d}$ satisfies one of the following equations:

$$
\int_{t_{d}(t)}^{0}\left(p(\Delta t)+p\left(|\Delta t|+2\left(t_{p}-t\right)\right)\right) d(\Delta t)=\Delta P \quad\left(t_{1}<t<t_{p}\right)
$$

or

$$
\int_{0}^{t_{d}(t)}\left(p(\Delta t)+p\left(-|\Delta t|+2\left(t_{p}-t\right)\right)\right) d(\Delta t)=\Delta P \quad\left(t_{p}<t<t_{2}\right)
$$

If the probability distribution of the timing jitter is known, then the equivalent time 
shifts can be calculated from eqs. (1.4) - (1.7), and the Markov estimator, $Y^{\prime}(t)$ can be compensated by an amount $\Delta Y(t)$ to yield an improved estimate, $Y^{\prime \prime}(t)$, as follow:

$$
\begin{array}{ll}
Y^{\prime \prime}(t)=Y^{\prime}(t)+\Delta Y(t) & \left(t_{1}<t<t_{2}\right) \\
\Delta Y(t)=Y^{\prime}\left(t-t_{d}(t)\right)-Y^{\prime}(t) & \left(t_{1}<t<t_{p}\right) \\
\Delta Y(t)=Y^{\prime}\left(t+t_{d}(t)\right)-Y^{\prime}(t) & \left(t_{p}<t<t_{2}\right)
\end{array}
$$

When $Y(t)$ is desired, we estimate $Y^{\prime}\left(t \pm t_{d}(t)\right)$ instead.

As a practical matter, the critical points, $t_{p}$, which appear in eqs. (1.4)-(1.7), must be determined from the Markov estimator, which will still contain some residual noise. The effects of the noise on the critical point determination can be minimized by using a multipoint discrete difference equation.

\section{APPROXIMATION, UP AND DOWN IN GENERALIZED FORM, NEW METHOD}

In this thesis we solve this problem from a different point of view and estimate the waveform by a curve instead of a point (median) as was done in the previous literatures. This curve is constructed from the percentiles of the sample distribution, whereas the Markov estimator and the Markov estimator with compensation are based on estimating the median. From simulation we know that median is not always the most efficient point to be used in Up and Down Method. No matter in consideration of the time or accuracy. This fact motivates us to use percentile as the estimator for the waveform.

Thus far, the problem has been changed to how to get an accurate estimate of the percentile of an unknown distribution. For estimating distribution-free percentiles in the real time sense, there are two methods available. One is Stochastic Approximation Method [5] and the other is Stochastic Up and Down Method in generalized form [6]. In addition to the above two methods, we propose a new method. Simulation results show 
that "Stochastic Up and Down Method in Generalized Form" and "The New Method" can achieve almost equal accuracy and are superior to the "Stochastic Approximation Method". In estimating a percentile of percentage less or greater than $50 \%$, "New Method" supersedes the "Stochastic Up and Down Method in Generalized Form" by having less sampling operations, which is equivalent to mechanical operations in disk drive. Electronic operations are much faster than the mechanical ones. The new method can achieve a more accurate estimated value, where "Stochastic Up and Down Method" has high bias, and vice versa. Also, when hardware implementation is concerned, "New Method" is simple in design. It is still an unsolved problem to optimize the parameter of "Stochastic Up and Down Method in Generalized Form" and the "New Method". 


\section{CHAPTER II}

\section{STOCHASTIC APPROXIMATION METHOD}

The Stochastic Approximation Method is concerned with schemes converging to some sought value when, due to the stochastic nature of the problem, observations involve errors. The interesting schemes are those which are self-correcting, that is, in which a mistake always tends to be corrected in the limit, and in which the convergence to the desired value is of some specified nature, for example, it is mean-squared convergent. The method in this section is a typical example of such a scheme for approximating, under suitable condition, the point where a regression function assumes a given value.

Let $F(x)$ be an unknown distribution function such that

$$
F^{\prime}(\theta)>0 \text {, for every } \theta \text { which makes } 0<F(\theta)<1 \text {. }
$$

and let $Z_{n}$ be a sequence of independent random variables each with the distribution function $\operatorname{Pr}\left\{Z_{n} \leq x\right\}=F(x)$. On the basis of $Z_{n}$ we wish to estimate $\theta$. However, as sometimes happens in practice ( bioassay, sensitive data ), we are not allowed to know the values of $Z_{n}$ themselves. Instead, we are free to prescribe for each $n$ a value $X_{n}$ and are then given only the value $y_{n}$ where

$$
y_{n}=\left\{\begin{array}{lll}
1 & \text { if } Z_{n} \leq X_{n} & \text { (response ) } \\
0 & \text { otherwise (no response) }
\end{array}\right.
$$

The convention for choosing $X_{n}$ proceeds as follows. Choose $X_{n}$ as our best guess of the value and $\left\{a_{n}\right\}$ be any sequence of constants of type $\frac{1}{n}$, which is defined below Definition 1 [5] 
Any sequence $\left\{a_{n}\right\}$ of positive constants satisfying

(1) $0<\sum_{1}^{\infty} \mathrm{a}_{\mathrm{n}}^{2}<\infty$

(2) $\sum_{n=2}^{\infty} \frac{a_{n}}{\left(a_{1}+\cdots+a_{n-1}\right)}=\infty$

is called sequence of type $\frac{1}{n}$.

Then obtain values $X_{2}, X_{3}, \cdots$ sequentially according to the rule

$$
X_{n+1}-X_{n}=a_{n}\left(\alpha-y_{n}\right) .
$$

It can be proved that [5]

$$
\lim _{n \rightarrow \infty} X_{n}=\theta
$$

$a_{n}$ in a looser definition is also studied in [7], [8]. In those condition this method also holds. Type $\frac{1}{n}$ sequence has served the practical purpose very well. When this is implemented, we choose $a_{n}=\frac{1}{n}$. Simulation results using both $X_{n}$ and $\frac{\sum X_{n}}{n}$ as an estimator of percentiles is shown in TABLE I and TABLE II. Even though the bias for percentile $5 \%$ to $50 \%$ in TABLE II seem to be small, there is difficulty in implementing this method to instrument or in general practice. The Stochastic Approximation Method is not appropriate for our problem. It requires the measuring instrument to assume values on almost the entire real line. It is not the case generally in practice. The instrument will usually be restricted to values on some lattice, e.g. $\{a+k \delta, k \in I\}$ for some index set $I$ [9]. However, in our simulation, we assume the probing instrument can take on the values on the whole real line and the numerical results are just for reference. 
TABLE I

PERCENTILE ESTIMATION

WITH DIFFERENT METHODS ON GAUSSIAN DISTRIBUTION REPETITIVE TIMES $10^{7}$

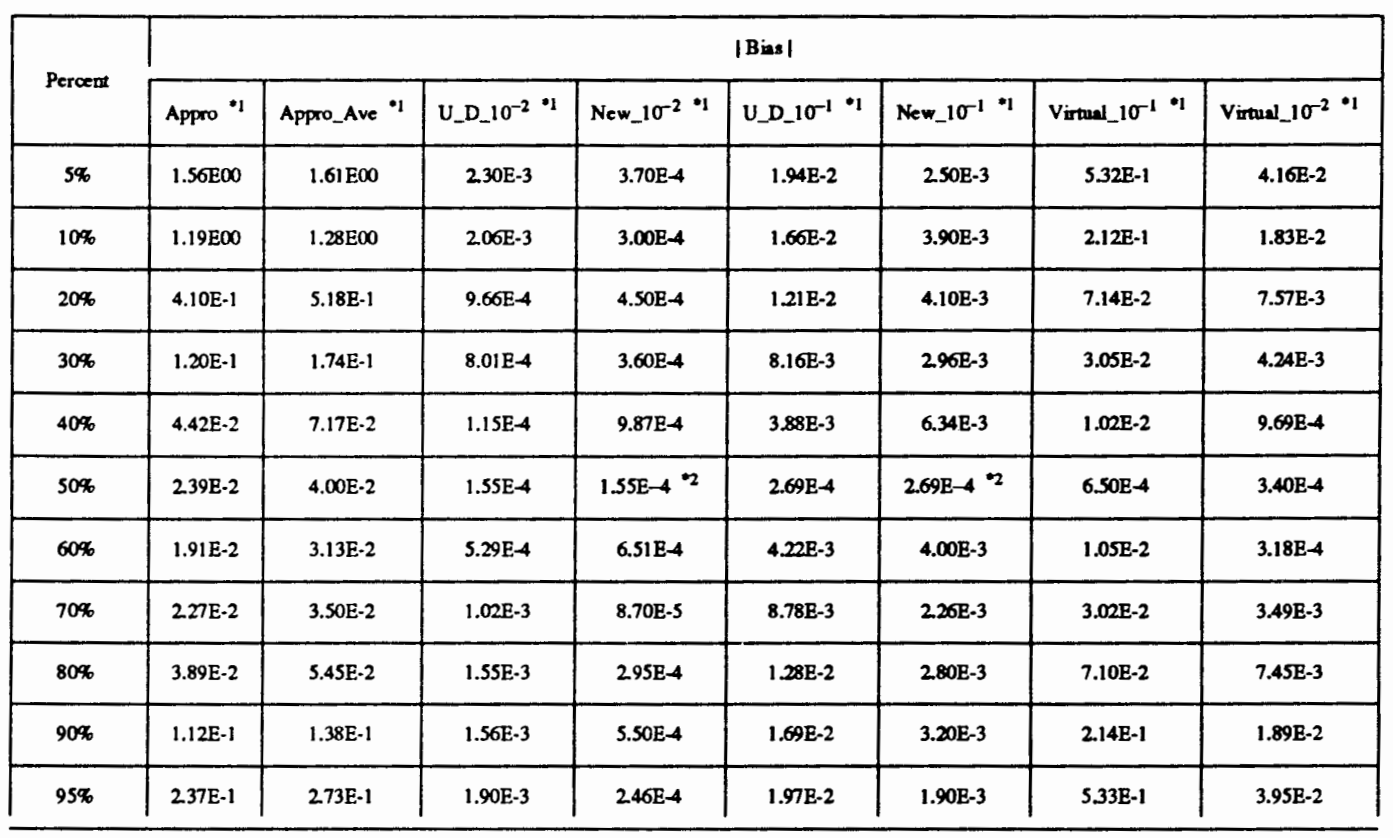

$* 1$

Appro : Stochastic Approximation Method an $=\frac{1}{n}$

Appro_Ave : Stochastic Approximation Method with Average of $x_{n}$ as Estimator

U_D_10 : : Scochastic Up and Dowe Method with $\delta=10^{-2}$

New_1 $10^{-2} \quad$ New Method with $\delta=10^{-2}$

U_D_1 $10^{-1}$; Stochastic Up and Dowo Method with $\delta=1 \sigma^{\prime}$

New_10 $10^{-1} \quad$ New Method with $\delta=10^{-1}$

Virtual_1 $10^{-1}$ : Virtual Prabability Method with $\delta=10^{-1}$

Virtual_1 $10^{-2}$ : Virtual Probability Method with $\delta=10^{-2}$

$\bullet 2$

In estimating $50 \%$ percentile new method has the same corvention as the Up and Down 
TABLE II

PERCENTILE ESTIMATION

WITH DIFFERENT METHODS ON EXPONENTIAL DISTRIBUTION REPETITIVE TIMES $10^{7}$

\begin{tabular}{|c|c|c|c|c|c|c|c|c|}
\hline Percemt & Appro $* 1$ & Appro_Ave ${ }^{* 1}$ & U_D_1 $10^{-2}$ & $\mathrm{New} w_{-} 10^{-2} \cdot 1$ & U_D_10 $10^{-1} \cdot 1$ & New_1 $\sigma^{-1} \cdot 1$ & Virtual_ $10^{-1}$ & Virtual_1 $1 \sigma^{-2} \cdot 1$ \\
\hline $5 \%$ & S.50E-5 & $1.34 \mathrm{E}-4$ & $2.14 \mathrm{E}-4$ & $5.76 \mathrm{E}-3$ & $3.96 \mathrm{E}-3$ & $3.11 \mathrm{E}-3$ & $6.80 \mathrm{E}-1$ & $3.15 \mathrm{E}-2$ \\
\hline $10 \%$ & $9.40 \mathrm{E} \cdot 5$ & $282 \mathrm{E} \cdot 5$ & $3.07 \mathrm{E}-4$ & $6.40 \mathrm{E}-3$ & $1.21 \mathrm{E}-3$ & $3.41 \mathrm{E}-2$ & $2.10 \mathrm{E}-1$ & $1.40 \mathrm{E}-3$ \\
\hline $20 \%$ & $248 \mathrm{E}-4$ & $1.97 \mathrm{E}-4$ & $7.27 \mathrm{E}-4$ & $7.90 \mathrm{E}-3$ & $5.17 \mathrm{E}-3$ & $6.35 \mathrm{E}-2$ & $1.65 \mathrm{E}-2$ & $2.10 \mathrm{E}-3$ \\
\hline $30 \%$ & $1.39 \mathrm{E}-4$ & $1.74 \mathrm{E}-4$ & $1.04 \mathrm{E}-3$ & $9.11 \mathrm{E}-3$ & $7.78 \mathrm{E}-3$ & $7.80 \mathrm{E}-2$ & $1.93 \mathrm{E}-2$ & $249 \mathrm{E}-3$ \\
\hline $40 \%$ & 8.97E-5 & $2.60 \mathrm{E} \cdot 5$ & $1.17 \mathrm{E}-3$ & $5.30 \mathrm{E}-3$ & $1.02 E-2$ & $4.89 \mathrm{E}-2$ & $2.49 \mathrm{E}-2$ & $2.28 \mathrm{E}-3$ \\
\hline $70 \%$ & $9.46 \mathrm{E}-3$ & $1.37 \mathrm{E}-2$ & $1.26 \mathrm{E}-3$ & $7.55 \mathrm{E}-4$ & $1.72 \mathrm{E}-2$ & $280 \mathrm{E}-3$ & $5.92 \mathrm{E}-2$ & $5.59 \mathrm{E}-3$ \\
\hline $80 \%$ & $2.98 \mathrm{E}-2$ & $3.80 \mathrm{E}-2$ & $1.62 \mathrm{E}-3$ & $6.80 \mathrm{E}-4$ & $1.95 \mathrm{E}-2$ & $3.60 \mathrm{E}-3$ & $1.05 \mathrm{E}-1$ & $1.16 \mathrm{E}-2$ \\
\hline $90 \%$ & $1.90 \mathrm{E}-1$ & $2.16 E-1$ & $1.71 \mathrm{E}-3$ & $4.70 \mathrm{E}-4$ & $2.90 \mathrm{E}-2$ & $2.21 \mathrm{E}-3$ & 2.45E-1 & $2.61 \mathrm{E}-2$ \\
\hline $95 \%$ & $5.30 \mathrm{E}-1$ & $5.73 \mathrm{E}-1$ & $207 \mathrm{E}-3$ & $1.90 \mathrm{E}-4$ & $2.31 \mathrm{E}-2$ & $8.29 \mathrm{E}-4$ & $5.50 \mathrm{E}-1$ & $4.90 \mathrm{E}-2$ \\
\hline
\end{tabular}

$* 1$

Appro : Stochastic Approx imation Method an $=\frac{1}{0}$

Appro_Ave : Stochastic Approximation Method with Average of $x_{n}$ as Estimator

U_D_10 : Stochastic Up and Down Method with $\delta=10^{-2}$

New_1 $10^{-2}:$ New Method with $\delta=10^{-2}$

U_D_10 : : Stochastic Up and Down Method with $\delta=10^{-1}$

New_10 $10^{-1} \quad$ New Method with $\delta=10^{-1}$

Virual_1 $1 \sigma^{-1}$ : Virtual Probability Method with $\delta=1 \sigma^{-1}$

Virtual_1 $1 \sigma^{-2}$ : Virtual Probability Method with $\delta=10^{-2}$

$* 2$

In estimating $50 \%$ percentile new method has the same convention as the UP and Down. 


\section{CHAPTER III}

\section{STOCHASTIC UP AND DOWN METHOD IN GENERALIZED FORM}

\section{CONVENTION}

This method is a generalized form of a Markov estimator. It is different from the Stochastic Approximation in two aspects even though they seem to be the same. One difference is the using of a fixed constant instead of $a_{n}$, which is a sequence of type $\frac{1}{n}$. The other is using

$$
\underbrace{\sum_{n}^{n} Y_{k}}_{k=1}
$$

as an estimator of a percentile instead of $X_{n}$.

Definition 2. Generalized Up and Down Method ( GUDM)

Determine a Markov Chain from quantal response data obtained in the observation of the trial. The underlying distribution function of the trial is $F$. Let $\mu_{p}$ be the percentile of $F$. An observation is a response or nonresponse depending on whether the value of the random variable with distribution $\mathrm{F}$ is less than or greater than some known value. If

$$
L=\left\{\ldots, \mathrm{d}_{1}, \mathrm{~d}_{0}, \mathrm{~d}_{1}, \ldots\right\}
$$

is a set of equally spaced levels with interval $\delta$, the method to be studied starts with a value $\varepsilon$ and continues for an additional $n-1$ trials as levels $Y_{2}, \ldots, Y_{n}$ determined by

$$
Y_{v+1}=\left\{\begin{array}{l}
Y_{v}+d \uparrow \text { if } X_{v} \geq Y_{v} \\
Y_{v}-d \downarrow \text { if } X_{v}<Y_{v}
\end{array}\right.
$$


, where $\frac{d \uparrow}{d \downarrow}=\frac{p \delta}{(1-p) \delta}=\frac{p}{1-p}, 0<p<1$

$\bar{Y}=\sum_{v=1}^{n} \frac{Y_{v}}{n}$ is used as an estimator of $\mu_{p}$ of $F$.

Simulation results on Exponential and Gaussian distributions are shown in TABLE I and TABLE II. The bias using $\delta=10^{-2}$ is generally smaller than that using $\delta=10^{-1}$. The former is one order less than the latter. The smallest bias in this simulation is at the 5\% percentile. The largest is at the 95\% percentile. In estimating the Exponential distribution, it is easy to recognize that when the percent of the percentile increases, the bias increases. In the Gaussian distribution there is greater bias as the percent of the estimated percentile deviates further from $50 \%$. All the methods we mention in this thesis, except the New Method, have this tendency. There is situation that we need to utilize the estimation of those high-biased percentiles [10]. In chapter IV, we propose the New Method to reduce the bias. What we achieve in the New Method is a procedure providing preliminary models to estimate the percentile of any distribution. The bias is kept low, compared with the other methods, to at least one side. We are using this characteristic to solve the jitter noise problem more efficiently when additive noise also exists.

\section{SOME PROPERTIES OF STOCHASTIC UP AND DOWN METHOD}

This section focuses on the theory aspect of the Up and Down Method in the ideal situation, that is $\delta \rightarrow 0, n \rightarrow \infty$. Some phenomena of this method when $n \rightarrow \infty, \delta \rightarrow 0$ are investigated. The discussion here is not concerned about the convergence of this method, but rather attribute to [3]. 
Definition 3 Inverse Up and Down Method ( IUDM )

Determine a Markov Chain from the quantal response data obtained in the observation of the trial. The underlying distribution of the trial is F. An observation is a response or nonresponse depending on whether the value of the random variable with distribution $F$ is less than or greater than some known value. If

$$
L=\left\{\ldots, d_{-1}, d_{0}, d_{1}, \ldots\right\}
$$

is a set of equally spaced levels with interval $\delta$, the method to be studied starts with a value $\varepsilon$ that is greater or less than median and continues for an additional $n-1$ trials as levels $Y_{2}, \ldots, Y_{n}$ determined by

$$
Y_{v+1}=\left\{\begin{array}{l}
Y_{v}-d \text { if } X_{v} \geq Y_{v} \\
Y_{v}+d \text { if } X_{v}<Y_{v}
\end{array}\right.
$$

\section{Assertion 1}

IUDM diverges, when $\delta \rightarrow 0, \mathrm{n} \rightarrow \infty$.

(i) If $\varepsilon>$ median of $F$, then $Y_{n} \rightarrow \infty$ and $Y_{n} \neq$ median of $F$ for all $n$.

(ii) If $\varepsilon<$ median of $F$, then $Y_{n} \rightarrow-\infty$ and $Y_{n} \neq$ median of $F$ for all $n$.

\section{Discussion}

To make the expression meaningful, we specify the approaching speed of $\delta$ to 0 as $\frac{1}{n^{q}}, 0<q<1$. Choose $\delta=\frac{1}{\sqrt{n}}$ randomly.

Case $1: \varepsilon>0$

To let $\mathrm{Y}_{\mathrm{k}}<\left(\frac{\varepsilon-\text { median }}{2}+\right.$ median $)<\mathrm{Y}_{\mathrm{k}+1}$ for some level number $\mathrm{k}$, we need at least $\mathrm{N}$ observations with nonresponse. $\mathrm{N}$ is calculated as below: 


$$
\begin{aligned}
\lim _{\delta \rightarrow 0} N & =\lim _{\delta \rightarrow 0} \frac{\varepsilon-\left(\frac{\varepsilon-\text { median }}{2}+\text { median }\right)}{\delta} \\
& =\lim _{\delta \rightarrow 0} \frac{\varepsilon+3 \times \text { median }}{\delta} \\
& =\infty .
\end{aligned}
$$

However, $\mathrm{P}\left\{\mathrm{X}>\frac{\varepsilon}{2}\right\}<\mathrm{P}\left\{\mathrm{X}<\frac{\varepsilon}{2}\right\}$ so we can conclude that

$$
P\left\{Y_{n}=\frac{\varepsilon}{2}, \text { for any } n\right\}=0
$$

Dividing $\mathrm{n}$ by a positive integer $\mathrm{k}$, we get

$$
\mathrm{n}=\mathrm{k} \mathrm{N}_{0}+\mathrm{n}_{0}^{\prime}, 0 \leq \mathrm{n}_{0}^{\prime}<\mathrm{k}
$$

Subsequently,

$$
Y_{n}=\varepsilon+\left(f_{1} N_{0} \delta+f_{2} N_{0} \delta+\cdots+f_{k} N_{0} \delta\right)+g n_{0}^{\prime} \delta
$$

where $f_{i}$ is the net fraction of $i$-th $N_{0}$ that contributes to the increase of $\varepsilon ; g$ is for $n_{0}^{\prime}$. When $n \rightarrow \infty, N_{0}=\frac{n-n_{0}^{\prime}}{k} \rightarrow \infty$. Also $0<f_{i}<1$ for $i=1$ to $k, f_{i} \leq f_{i+1}$, for $i=1$ to $k$ - 1 ; and $g$ is the net fraction of $n_{0}$ that contribute the increase of $\varepsilon$. We note that $g$ could be a negative number.

So that,

$$
\begin{aligned}
Y_{n} & \geq \varepsilon+\left(f_{1}+\cdots+f_{k}\right) N_{0} \delta \\
& \geq \varepsilon+k_{1} N_{0} \delta
\end{aligned}
$$

As a result,

$$
\lim _{n \rightarrow \infty} Y_{n}>\lim _{n \rightarrow \infty}\left(\varepsilon+k f_{1} \frac{N_{0}}{\sqrt{n}}\right)=\lim _{n \rightarrow \infty}\left(\varepsilon+k f_{1} \frac{n-n_{0}^{\prime}}{k} \frac{1}{\sqrt{n}}\right)=\infty
$$

Case 2: $\varepsilon<0$

Argument for case 2 are similar to case 1 . 
Corollary 1.1

$F(D)=1, \varepsilon>$ median By the IUDM, $Y_{n}$ is approaching $\infty$. Assume $Y_{p} \geq D, Y_{p-1}<D$. When level $p$ is reached, we adopt the convention of SUDM. $Y_{n}$ will finally reach $\varepsilon$, and $Y_{n} \neq$ median for every $n$

Corollary 1.2

$F(D)=0, \varepsilon<$ median By the IUDM, $Y_{n}$ is approaching $-\infty$. Assume $Y_{p} \geq D, Y_{p-1}>D$ . When level $p$ is reached, we adopt the convention of SUDM. $Y_{n}$ will finally reach $\varepsilon$, and $Y_{n} \neq$ median for every $n$

\section{Corollary 1.3}

When $\mathrm{n} \rightarrow \infty, \delta \rightarrow 0$ with convergence speed of type $\frac{1}{\mathrm{n}^{\mathrm{k}}}, 0<\mathrm{k}<1$, then Up and Down Method will generate an infinite sequence with monotonic increasing values toward the median by selecting the level values appearing the first time as the elements of the sequence.

Discussion:

As proved in [3], when, $\mathrm{n} \rightarrow \infty, \delta \rightarrow 0$ with convergence type $\frac{1}{\mathrm{n}^{\mathrm{k}}}, 0.5 \leq \mathrm{k}<1$ then this infinite sequence converges to the median with probability 1 . We can conclude $Y_{n}$ will not exceed the median in this situation.

Definition 4 Inverse Generalized Up and Down Method ( IGUDM)

All the symbols are defined in Definition 1.

$$
Y_{v+1}=\left\{\begin{array}{l}
Y_{v}-d \uparrow \text { if } X_{v} \geq Y_{v} \\
Y_{v}+d \downarrow \text { if } X_{v}<Y_{v}
\end{array}\right.
$$


Assertion 2

IGUDM diverges when $\delta \rightarrow 0, \mathrm{n} \rightarrow \infty$.

(i) If $\varepsilon>\mu_{\mathrm{p}}$, then $\mathrm{Y}_{\mathrm{n}} \rightarrow \infty, \mathrm{Y}_{\mathrm{n}} \neq \mu_{\mathrm{p}}$ for all $\mathrm{n}$.

(ii) If $\varepsilon<\mu_{\mathrm{p}}$, then $\mathrm{Y}_{\mathrm{n}} \rightarrow-\infty, \mathrm{Y}_{\mathrm{n}} \neq \mu_{\mathrm{p}}$ for all $\mathrm{n}$.

Discussion:

To make the assertion meaningful, we specify the approaching speed of $\delta \downarrow$ to 0 as

$\frac{1}{n^{q}}, 0<q<1$. Choose $\delta \downarrow=\frac{1}{\sqrt{n}}, n \rightarrow \infty$ randomly.

Case $1: \varepsilon>\mu_{\mathrm{p}}$

To let $\mathrm{Y}_{\mathrm{k}}<\left(\frac{\varepsilon-\mu_{\mathrm{p}}}{2}+\mu_{\mathrm{p}}\right)<\mathrm{Y}_{\mathrm{k}+1}$ for some level number $\mathrm{k}$, we need at least $\mathrm{N}$ observations with $\mathrm{n}$ response. $\mathrm{N}$ is calculated below:

$$
\begin{aligned}
\lim _{\delta \rightarrow 0} N & =\lim _{\delta \rightarrow 0} \frac{\varepsilon-\left(\frac{\varepsilon-\mu_{\mathrm{p}}}{2}+\mu_{\mathrm{p}}\right)}{\delta} \\
& =\lim _{\delta \rightarrow 0} \frac{\varepsilon+3 \mu_{\mathrm{p}}}{\delta}=\infty
\end{aligned}
$$

However,

$$
\begin{aligned}
& \mathrm{P}\left\{\mathrm{X}>\frac{\varepsilon-\mu_{\mathrm{p}}}{2}+\mu_{\mathrm{p}}\right\}<\mathrm{P}\left\{\mathrm{X}<\frac{\varepsilon-\mu_{\mathrm{p}}}{2}+\mu_{\mathrm{p}}\right\} \text { for } \mu_{\mathrm{p}}>0.5 \\
& \mathrm{P}\left\{\mathrm{X}>\frac{\varepsilon-\mu_{\mathrm{p}}}{2}+\mu_{\mathrm{p}}\right\}>\mathrm{P}\left\{\mathrm{X}<\frac{\varepsilon-\mu_{\mathrm{p}}}{2}+\mu_{\mathrm{p}}\right\} \text { for } \mu_{\mathrm{p}}<0.5
\end{aligned}
$$

So, we can conclude that $\mathrm{P}\left\{\mathrm{Y}_{\mathrm{N}} \geq \mu_{\mathrm{p}}\right\}=1$. Divide $\mathbf{n}$ by a positive number $\mathbf{k}$, we get

$$
\mathrm{n}=\mathrm{k} \mathrm{N}_{0}+\mathrm{n}_{0}^{\prime}, 0 \leq \mathrm{n}_{0}^{\prime} \leq \mathrm{k}
$$

Subsequently,

$$
Y_{n}=\varepsilon+\left(f_{1} N_{0} \delta \uparrow+f_{2} N_{0} \delta \uparrow+\cdots+f_{k} N_{0} \delta \uparrow\right)+g n_{0}^{\prime} \delta \uparrow
$$

where $f_{i}$ is the net fraction of $i$-th $N_{0}$ that contributes to the increase of $\varepsilon ; g$ is for $n_{0}^{\prime}$; When $n \rightarrow \infty, N_{0}=\frac{n-n_{0}^{\prime}}{k} \rightarrow \infty$. Also $0<f_{i} \leq 1$ for $i=1$ to $k, f_{i} \leq f_{i+1}$, for $i=1$ to 
$\mathrm{k}-1$; And $\mathrm{g}$ is the net fraction of $\mathrm{n}_{0}$ that contributes the increase of the level value. Again $\mathrm{g}$ could be a negative number.

So that

$$
\begin{aligned}
\mathrm{Y}_{\mathrm{n}} & \geq \varepsilon+\left(\mathrm{f}_{1}+\cdots+\mathrm{f}_{\mathrm{k}}\right) \mathrm{N}_{0} \delta \uparrow \\
& >\varepsilon+\mathrm{kf}_{1} \mathrm{~N}_{0} \delta \uparrow
\end{aligned}
$$

As a result,

$$
\lim _{n \rightarrow \infty} Y_{n}>\lim _{n \rightarrow \infty}\left(\varepsilon+k f_{1} \frac{N_{0} p}{\sqrt{n}}\right)=\lim _{n \rightarrow \infty}\left(\varepsilon+k f_{1} \frac{n-n_{0}^{\prime}}{k} \frac{p}{\sqrt{n}}\right)=\infty
$$

Case 2: $\varepsilon<\mu_{\mathrm{p}}$

Arguments for case 2 are similar to case 1 .

Corollary 4.1

Suppose $F(D)=1, \varepsilon>\mu_{p}$ By IUDM, $D$ is on the way to $\infty$. Assume $Y_{p} \geq D, Y_{p-1}<D$. When level $p$ is reached, we adopt the convention of SUDM. $Y_{n}$ will finally reach $\varepsilon$, and $Y_{n} \neq \mu_{p}$ for every $n$

Corollary 4.2

$F(D)=0, \varepsilon<\mu_{p}$. $Y_{n}$ is approaching $-\infty$. Assume $Y_{p} \leq D, Y_{p-1}>D$. When level $p$ is reached, we adopt the convention of SUDM. $Y_{n}$ will finally reach $\varepsilon$, and $Y_{n} \neq \mu_{p}$ for every $n$

Corollary 4.3

When $\mathrm{n} \rightarrow \infty, \delta \rightarrow 0$ with convergence speed of type $\frac{1}{n^{k}}, 0<k<1$, Generalized Up and Down Method will generate an infinite sequence with monotonic increasing value toward $\mu_{\mathrm{p}}$ by selecting the level values appearing the first time as the elements of the sequence.

Discussion:

As proved in [3], when $\delta \rightarrow 0$ with convergence type $\frac{1}{n^{k}}, 0.5 \leq k<1$ this infinite 
sequence converge to $\mu_{p}$ with probability 1 . We can also conclude $Y_{n}$ will not exceed $\mu_{p}$ in this situation.

The problem of concentration of the stationary distribution of $Y_{n}, n \rightarrow \infty$ when estimating percentile is still under investigation. In this section as well as in [3], the study focuses on $\delta \rightarrow 0$, not a fixed small number. In fact, what we used in this chapter can be generalized further. $F(x)$ can be replaced by a function $M(x)$, which is the expectation value at level $x$. And then, this method is applied to find $\theta$ such that $M(\theta)=\alpha$, where $\alpha$ is given. Apparently, this is a more general regression problem. Using the notation, consider any random variable $\mathrm{Y}$ which is associated with an observable value $\mathrm{x}$ in such a way that the conditional distribution function of $Y$ for fixed $x$ is $H(y \mid x)$, the function $\mathrm{M}(\mathrm{x})$ is then the regression of $\mathrm{Y}$ on $\mathrm{x}$. 


\title{
CHAPTER IV
}

\author{
NEW METHOD
}

\section{VIRTUAL PROBABILITY}

Given a probability density function( PDF ). If we count on the quantal response from the PDF to carry out some procedure, we can manipulate the procedure without the reference of the quantal response and assume it is caused by a new PDF, which originates from the previous one. "Virtual part " is defined to be the probability of the new PDF generated from controlling the procedure. Create a virtual part with probability $\frac{2 P-1}{2 P}$ to make $\mu_{p}$ become the median of the new probability density function. If we use the same convention as Stochastic Up and Down Method with $\delta \uparrow=\delta \downarrow$ then both $\bar{Y}=\lim _{n \rightarrow \infty} \frac{\sum Y_{n}}{n}=\mu_{p}$ and $\lim _{n \rightarrow \infty} Y_{n}=\mu_{p}$ are proved [3]. We will use both $Y_{n}$ and $\bar{Y}$ as estimator to compare the accuracy. Associating $F(x)$ with a uniform random variable generator $(0,1)$, we can divide the interval into two parts $\left(0, \frac{1}{2 P}\right)$ and $\left(\frac{1}{2 P}, 1\right)$. Even though the convergence of this method is easy to see, it causes extra overhead when implemented into instruments. By breaking the virtual part into pieces, we devise the New Method in 4.2. 


\section{NEW METHOD - VIRTUALLY \\ GENERATE DYNAMICALLY SYMMETRIC P.D.F.}

The motivation of this method is by viewing that for the Stochastic Up and Down Method if the underlying distribution is symmetric about the median, then the stationary distribution of $Y_{n}, n \rightarrow \infty$ is almost symmetric about the median. So

$$
\left|M-\mu_{p}\right| \leq \frac{d}{2}
$$

Where $M$ is the expectation value of stationary distribution of $Y_{n}$, when $n \rightarrow \infty$ [11]. This method creates a definite portion of virtual response as a function of $p$. And suppose there is a mechanism, which is a black box, to manipulate these virtual response to create a symmetric P.D.F. about $\mu_{p}$. This mechanism can change the status of these virtual points when necessary. Another mechanism, which is also a black box, is an error counter to calculate the portion of virtual points with status changed. Although the operation of both mechanisms are unknown at the moment, it is expected to improve this method, which has gained the reliability from simulation, from these two components in the future study. Thus far, we have created two parallel computations: one is real implementation by Stochastic Up and Down for estimating the median, the other is a virtual implementation which generates a symmetric P.D.F. with the aid of black boxes. As we can see, in the virtual implementation, the stationary distribution of $Y_{n}, n \rightarrow \infty$ will concentrate to $\mu_{p}$, as $\delta$ decreases. This means less chance for virtual point to change status. Then the equivalent underlying distribution in real implementation is more similar to the symmetric P.D.F., so $\bar{Y}$ is closer to $\mu_{p}$. We are attempting to prove in the virtual implementation the existence of stationary distribution of $Y_{n}, n \rightarrow \infty$, when $\delta \neq 0$.

This method does not work very well in those levels that are difficult for virtual points to maintain the status. An example, is when calculating the percentile of percentage less than 50\% percentile in the Exponential distribution. The characteristics of these 
levels are their locations in high probability density.

The rules of this method are shown in subsequent paragraphs. The purpose of these rules are to mix the virtual points to the original stochastic process as naturally as possible. At the moment, there is no strict theoretical discussion about these rules. They are devised empirically. However, it is clear that the study about the theoretical part will contain the statistics, which is concerned about the ensemble of the process, and combinatorics, which are related to the individual effect of each stage in the process. Three different kinds of points will be created through these rules. Each point represents a stage in the process. They are normal, virtual, stablizing.

\section{Definition 5}

Normal points represent the samples appearing in the original PDF. They follow the original stochastic process to add $\delta$ or subtract $\delta$.

\section{Definition 6}

Virtual points represent the samples appearing in the virtual portion. They force the increment or increment of level value depending on the location of the virtual portion without carrying out the sampling.

\section{Definition 7}

Stablizing points are modified normal points. SN represents stablizing point for normal distribution; SE represents stablizing point for Exponential distribution. Before the operation of the normal points is initiated, they are forced to add or subtract the level according to the following rules:

(1) For estimating percentile greater than $50 \%$ :

Normal Distribution : 
+ means an added positive response.

$\mathrm{SN}$ follows the original stochastic process.

- means an added negative response.

They act in order.

Exponential Distribution :

$-\mathrm{SE}+$

,+- have the same definition as above.

SE follows the original stochastic process. And exports the sampling value to the next normal point.

They act in order.

(2) For estimating percentile less than 50\%:

Normal Distribution :

$-\mathrm{SN}+$

,+- have the same definition as above.

SN follows the original stochastic process.

They act in order.

Exponential Distribution :

$+\mathrm{SE}$ -

,+- have the same definition as above.

SE follows the original stochastic process. And exports the sampling value to the next normal point. 
They act in order.

\section{Definition 8.}

If $0 . d_{1} d_{2} \cdots d_{n}$-percentile is the unknown, then

$$
\begin{aligned}
\text { left number } & =d_{1} \times 10^{n-1}+d_{2} \times 10^{n-2}+\cdots+d_{n} \\
\text { right number } & =10^{n}-d_{1} \times 10^{n-1}+d_{2} \times 10^{n-2}+\cdots+d_{n} .
\end{aligned}
$$

$d_{i}$ is integer ranging from $0 . . .9$.

\section{Definition 9.}

number of virtual point $=\mid$ left number - right number $\mid$.

Definition 10.

If $\frac{\text { left number }}{\text { right number }}>2$ then number of stablizing point $=$ left number - right number

else number of stablizing point $=\frac{\text { number of virtual point }}{2}$

Definition 11.

cycle number $=$ left number + right number + number of virtual point

It sets the status of virtual or stablizing point in a cycle controlled by a cycle number. And points with different status have their own operation respectively as defined in Definition 7.

We are now ready to discuss the new algorithm as follow :

\section{Algorithm}

Input : left number( or right number)

Output : the status of each point and its operation

REPEAT $\left\{\mathrm{n} \_\right.$int $=1$... cycle number. $\}$ 
IF $n \_$int is an even number

\{ This decision can be made by assign operation instead of division. \}

THEN even_count=even_count+1

IF $n \_$int is an even number AND even_count $>$

left number( or right number)

number of virtual point + number of stablizing point

THEN

BEGIN

IF stable_count $<$

$\frac{\text { number of virtual point }}{\text { number of stablizing point }}$

\section{THEN}

\{ It is a virtual point. \}

BEGIN

$\{$ Operation of virtual point ; refer to Definition 7$\}$

even_count $=$ even_count-

$\frac{\text { left number (or right number) }}{\text { number of virtual point }+ \text { number of stablizing point }}$

stable_count=stable_count +1

$n_{-}$int $=n_{-}$int +1

END

ELSE

$\{$ It is a stablizing point ; refer to Definition 7$\}$

BEGIN

\{Operation of stablizing point. \} 


$$
\begin{aligned}
& \text { even_count }=\text { even_count- } \\
& \frac{\text { left number }(\text { or right number })}{\text { number of virtual point }+ \text { number of stablizing point }} \\
& \text { stable_count }=\text { stable_count- } 1 \\
& \mathrm{n} \text { _int }=\mathrm{n} \text { _int }+1
\end{aligned}
$$

END

\section{END}

\section{ELSE}

\{ It is a normal point. \}

\section{BEGIN}

\{ Operation of normal point ; refer to Definition 7 \}

$$
\mathrm{n} \_ \text {int }=\mathrm{n} \_ \text {int }+1
$$

\section{END}

UNTIL_n_int $>$ cycle number.

This algorithm sets the status of virtual or stablizing point in a cycle controlled by a cycle number. And points with different status have their own operation respectively as defined in Definition 7. Even_count is the first criteria indicating when stablizing and virtual points will occur. Once the first requirement is met, stable_count acts as the second criteria to deciding it is a stablizing point or virtual point. The ratios in the algorithm are indicators for evenly spreading the virtual and stablizing points on the even points.

Repeatedly using the cycle set up previously to implement the basic Stochastic Up and Down Method $n \rightarrow \infty$ and $\delta \rightarrow 0$, the estimation of the New Method converges to the percentile in probability.

It is easy to see that $\delta$ has to change according to the range of the distribution. Before optimization is concerned in our study, we adopt rule of thumb to decide $\delta$ when 
conducting a fair comparison.

\section{Rule of Thumb}

Choose $\delta=0.1$ in normal distribution $\mathrm{N}(0,1)$ as template to get $\alpha=\frac{\delta}{\mu_{0.9999}-\mu_{0.0001}}=\frac{0.1}{2 \times 1.64} \alpha$ represents the relationship between $\delta$ and the range of the distribution. Use $\alpha$ as an reference when $\delta$ in the algorithm is concerned.

This algorithm is designed for Gaussian or Exponential distribution. For those distribution which are not standard Normal or Exponential, we use the algorithm for Gaussian distribution first and obtain three numerical data : $50 \%$ percentile, maximum value and minimum value. If $\mid$ maximum - $50 \%$ percentile $|>|$ minimum - $50 \%$ percentile $\mid$, then those percentiles for greater than or equal to $50 \%$ percent are retained. This is because in the New Method the longer side has lower bias when the underlying distribution is skewed to one side. For the rest of the percentiles, we linearly shorten the range of 50-percentile to maximum, so that the ratio $\frac{50 \% \text { percentile-minimum }}{\text { maximum }-50 \% \text { percentile }}>1$. Then apply the algorithm for normal distribution again. Under the calibration, the underlying distribution is skewed to the opposite side and those percentiles for less than $50 \%$ percent are retained. Similar operation apply to the situation when $\mid$ maximum - $50 \%$ percentile $\mid<1$ minimum - $50 \%$ percentilel is the initial condition. The other alternative is to obtain median, maximum, minimum value in preprocessing and then estimate the percentiles at the same time. In chapter $V$ the application in the waveform estimation with jitter noise shows us with satisfactory result compared with the Up and Down Method, even though the underlying distribution is not standard normal.

Simulation results are shown in TABLE 1, TABLE 2, Figure 3-6. If we adopt the 
general model in the estimation of percentiles for Gaussian and Exponential distribution, the bias is smaller than all the other methods in almost all the estimation. However, this seems to break the rule of a fair comparison. Before a clear choice is made for a fair comparison, we conclude with the fact that if we are allowed to choose any two values from the increment or decrement used by the Up and Down Method as the $\delta$ in the New Method, then the estimation is most satisfactory over the others.

One comment about the efficiency of the New Method compared with the others: with the utilization of virtual and stablized points, the sampling times needed have been reduced, which consumes most of the time. For percentiles between $33 \%$ and $67 \%$, sampling times are reduced $40 \%$. For other percentiles sampling times are reduced $50 \%$. If we adopt " New Method " to estimate the percentiles with high percentage in Exponential , low or high percentage in Gaussian or specified percentiles ( depicted in chapter V) in general distribution, one can obtain low bias with high efficiency.

From the data in TABLE I and TABLE II, we can recognize that "Up and Down" and "New Method" are the two with higher accuracy over the others. Furthermore, "New Method" has the lowest bias over all the other methods in estimating percentile of high percentage or low percentage. In estimating Exponential, Gaussian and general distribution its bias has the tendency of keeping low in at least one side. This is an useful characteristic when "New Method" is applied. Figure 3-6 exhibit the comparison between "Up and Down" and "New Method". The numerical values are from TABLE I and TABLE II. In estimating the Exponential distribution, as shown in Figure 3,4, New Method has higher bias than Up and Down Method in percentiles of percentage less than 50\%, and vice versa. At 50\%-percentile, both methods act in the same way. In estimating Gaussian distribution, as shown in Figure 5,6, the New Method has higher bias in estimating 30\% to $60 \%$ percentiles and vice versa. In estimating $50 \%$ percentile both methods act in the 
same way. One of the contribution of New Method is to reduce the bias shown in the figures.

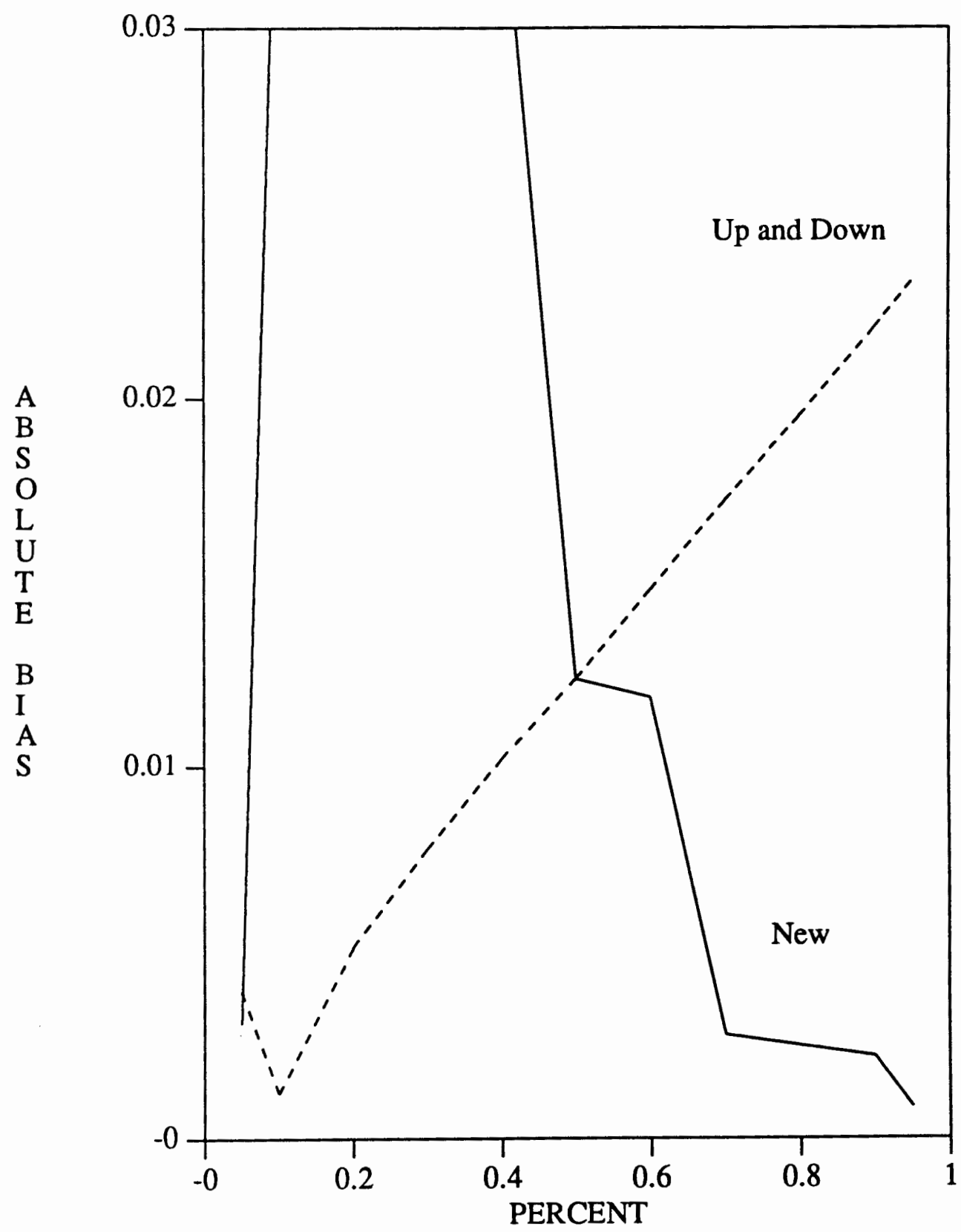

Figure 3. Bias of Percentile Estimation for Exponential Distribution $\mathrm{N}=10^{7}, \delta=10^{-1}$. 


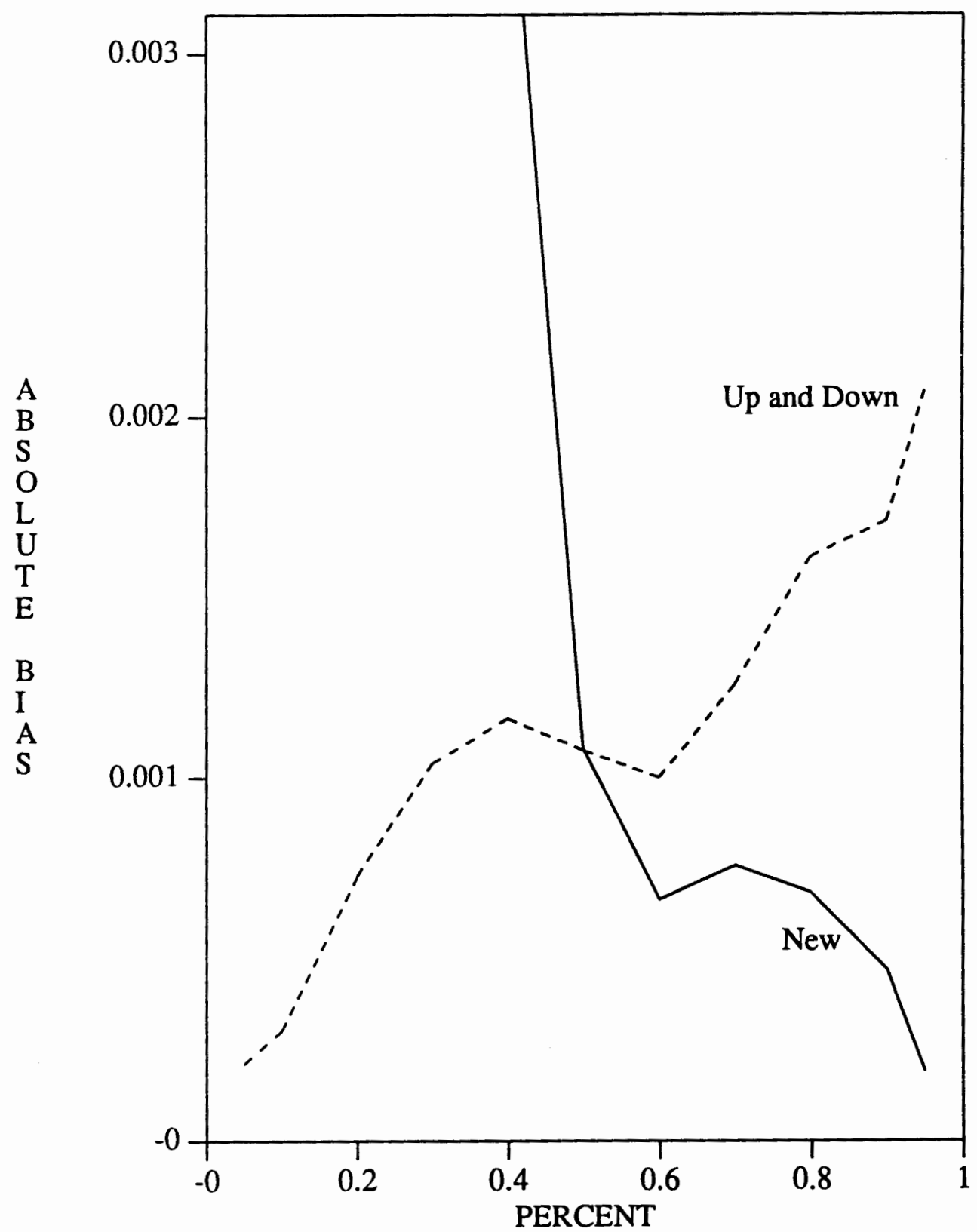

Figure 4. Bias of Percentile Estimation for Exponential Distribution $\mathrm{N}=10^{7}, \delta=10^{-2}$. 


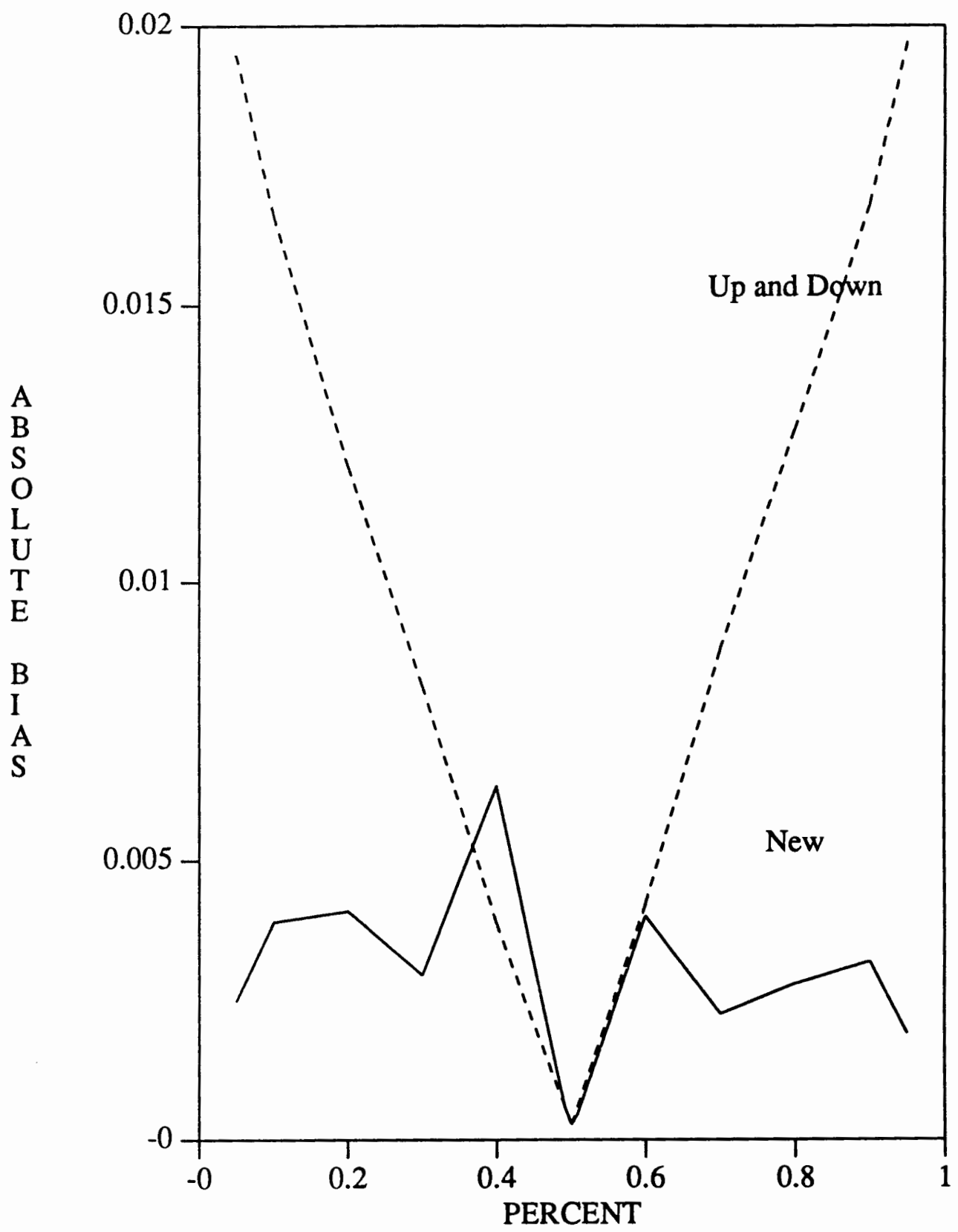

Figure 5. Bias of Percentile Estimation for Normal Distribution $\mathrm{N}=10^{7}, \delta=10^{-1}$. 


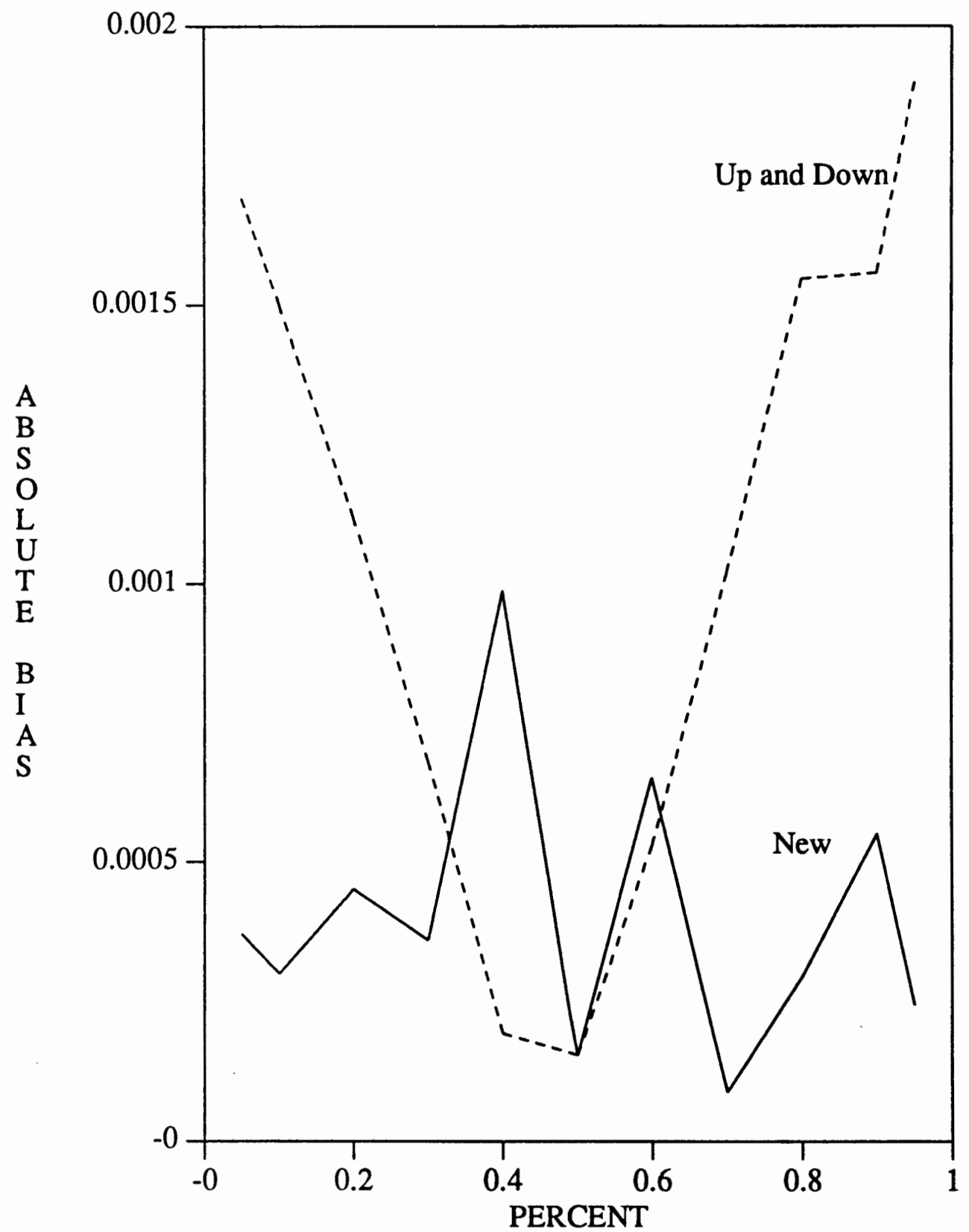

Figure 6. Bias of Percentile Estimation for Normal Distribution $\mathrm{N}=10^{7}, \delta=10^{-2}$. 


\section{CHAPTER V}

\section{APPLICATION}

Consider a waveform $y=\sin (0.1 \times t)-0.1 \times \sin (0.3 \times t)$ to be sampled near the peak. As illustrated in Figure 7.

The jitter distribution is Gaussian with mean 0 and standard deviation 1 modified to be finite support around the area 0.05 -percentile and 0.95 percentile. Jitter span is approximately $\frac{1}{10}$ of the period of the waveform.

The distribution is as follow :

TABLE III

DISTRIBUTION OF N(0,1)

MODIFIED TO BE FINITE SUPPORT AT \pm 1.8

\begin{tabular}{|c|c|}
\hline Percentage (\%) & Percentile \\
\hline 00.00 & -1.8 \\
\hline 06.68 & -1.5 \\
\hline 11.51 & -1.2 \\
\hline 18.41 & -0.9 \\
\hline 27.43 & -0.6 \\
\hline 38.21 & -0.3 \\
\hline 50.00 & 0.0 \\
\hline 61.79 & 0.3 \\
\hline 72.57 & 0.6 \\
\hline 81.59 & 0.9 \\
\hline 88.49 & 1.2 \\
\hline 93.32 & 1.5 \\
\hline 100.00 & 1.8 \\
\hline
\end{tabular}




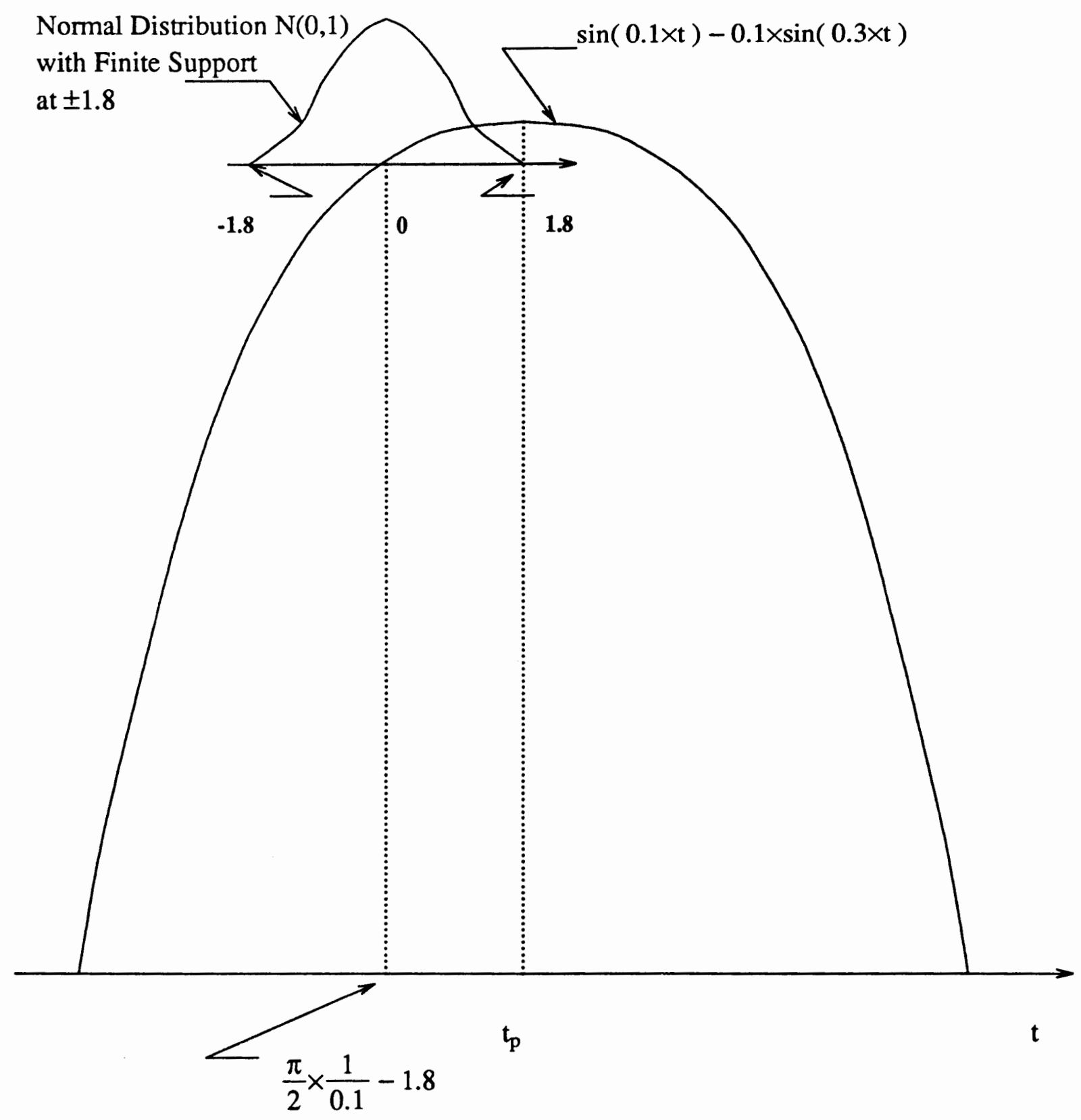

Figure 7. Sample $\sin (0.1 \times t)-0.1 \times \sin (0.3 \times t)$ with Jitter Distribution $N(0,1)$ Modified to be Finite Support at \pm 1.8

It is a practical matter to know $t_{p}$ at first. This can be done by the basic Stochastic Up and Down Method and reduce the noise of $t_{p}$ by using multiple point interpolation. Set $t=\frac{\pi}{2} \frac{1}{0.1}-1.8$, so that the jitter span is aligned with $t_{p}$. Then use Up and 
Down Method to find all the percentile listed in table in one stochastic process. Simulation results are in TABLE IV and Figure 8. At point 7, which is $50 \%$ percentile estimate, both methods have the same bias. It is because their operations are the same. Points 2-7 are the percentile estimates with percentage $6.68 \%$ to $38.21 \%$. New Method has lower bias than Up and Down Method. In this case, $\left|\mu_{0.05}-\mu_{0.5}\right|>\left|\mu_{0.99}-\mu_{0.5}\right|$, there is no need to calibrate the sampling value when estimating $\mu_{0.05}$ to $\mu_{0.5}$. However, we do need to calibrate the sampling value and choose a new $\delta$ according to Rule of Thumb. We have gained a good estimation for points 2-7 by using New Method.

TABLE IV

BIAS OF WAVEFORM ESTIMATION

FOR

$$
y=\sin (0.1 \times t)-0.1 \times \sin (0.3 \times t)
$$

\begin{tabular}{|c|c|c|}
\hline \multirow{2}{*}{ Point } & \multicolumn{2}{|c|}{ Bias } \\
\cline { 2 - 3 } & Up and Down & New for General \\
\hline 1 & 0.00 & 0.00 \\
\hline 2 & $-2.00 \mathrm{E}-4$ & $5.00 \mathrm{E}-5$ \\
\hline 3 & $-2.29 \mathrm{E}-4$ & $-3.09 \mathrm{E}-5$ \\
\hline 4 & $-1.78 \mathrm{E}-4$ & $-3.20 \mathrm{E}-5$ \\
\hline 5 & $-1.40 \mathrm{E}-4$ & $-6.10 \mathrm{E}-5$ \\
\hline 6 & $1.10 \mathrm{E}-4$ & $-7.00 \mathrm{E}-5$ \\
\hline 7 & $-7.60 \mathrm{E}-5$ & $-7.60 \mathrm{E}-5$ \\
\hline 8 & $-5.00 \mathrm{E}-5$ & $2.65 \mathrm{E}-5$ \\
\hline 9 & $-1.90 \mathrm{E}-5$ & $4.4 \mathrm{E}-5$ \\
\hline 10 & $-2.20 \mathrm{E}-5$ & $7.72 \mathrm{E}-5$ \\
\hline 11 & $-8.70 \mathrm{E}-5$ & $1.07 \mathrm{E}-4$ \\
\hline 12 & $-2.65 \mathrm{E}-4$ & $1.53 \mathrm{E}-4$ \\
\hline 13 & 0.00 & 0.00 \\
\hline
\end{tabular}




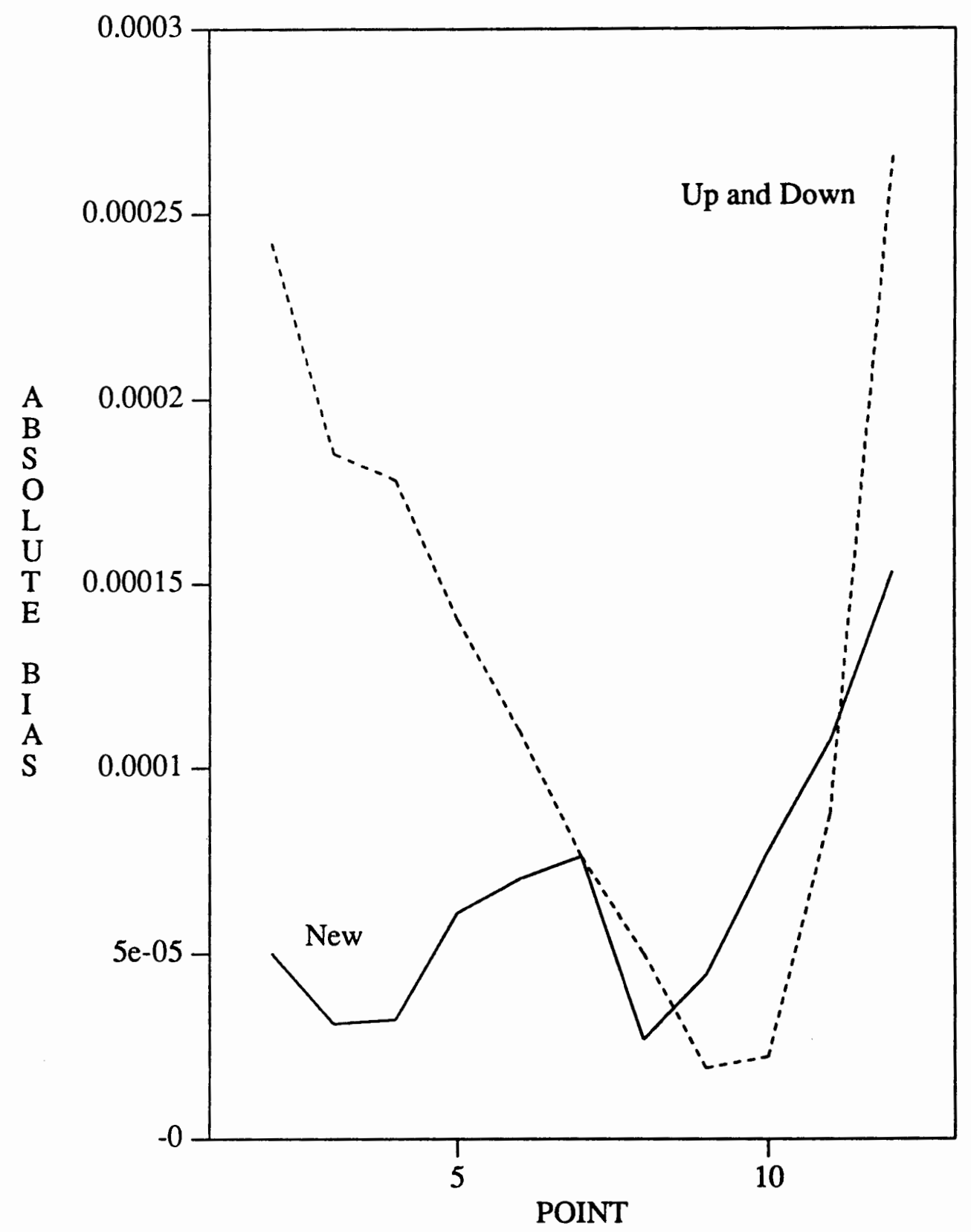

Figure 8. Bias of Waveform Estimation with Jitter Noise $\mathrm{N}=10^{7}$. 


\section{CHAPTER VI}

\section{CONCLUSION}

In the thesis we review methods to find the percentile of any distribution with the advantage of being distribution free. They are Stochastic Approximation Method, Stochastic Up and Down Method, and a proposed New Method --- Stochastic Up and Down by Pseudo Symmetrical Probability Density Function. The simulation result show that "Stochastic Up and Down by Pseudo Symmetry Probability Density Function" and "Stochastic Up and Down" are the two with more accurate results than Stochastic Approximation Method. Further, if we choose to estimate some specified percentiles, e.g. high percentage percentile in Exponential distribution etc., New Method can get low bias with reduced sampling times. However, there are several points to be solved. Otherwise, the applicability of these method are limited. We list them for future study.

1. Theoretical support of Stochastic Up and Down Method in generalized form. A proof in the ideal case is necessary.

2. Parameters $n$ and $\delta$ need to be specified in some particular way for effective performance.

3. The confidence interval of the results from these methods.

4. Estimation methods when both additive noise and jitter noise exist. 


\section{REFERENCES}

[1] W. L. Gans, "The Measurement and Deconvolution of Time Jitter in EquivalentTime Waveform Sampler," IEEE Transactions on Instrumentation and Measurement, Vol. IM-32, NO. 1. March 1983.

[2] Charles Hagwood, Grace L. Yang and T. M. Souders, "Weak Convergence of the Stochastic Up and Down Method", Private Communication.

[3] Y.C. Jenq and Wei-Da Hao, "Convergence in Probability of Up and Down Method", in preparation.

[4] G. Tong and T. M. Souders, "Compensation of Markov Estimator Errors in TimeJittered Samp ling of Non-monotonic Signals", Private Communication.

[5] H. Robbins and N. Carolina, "A Stochastic Approximation Method", Ann. Math. Stat. Vol 22, (1951).

[6] T. M. Souders, D. R. Flach, C. Hagwood and G. L. Yang, "The Effects of Timing Jitter in Sampling Systems", IEEE Transaction on Instrumentation and Measurement, Vol. 39, NO. 1, February 1990.

[7] J. Wolfowitz, "On the Stochastic Approximation Method of Robbin and Monro", Ann. Math. Stat. Vol. 23 (1952).

[8] A. Dvoretzky, "On Stochastic Approximation", Proc. of 3rd Berkeley Symposium on Math. Stat. \& Prob. Vol. 1 (1956).

[9] Charles Hagwood, T. Michael Souders, "A Discrete Stochastic Approximation Method with Applications to Estimation of Timing Jitter", Private Communication.

[10] Wei-Da Hao, "Waveform Reconstruction with Jitter Noise", in preparation.

[11] Brownlee, K. A., Hodges, J. L., Jr., and Rosenblatt, M., "The up-and-down method with small samples", J. of the American Statistical Association, 48 (1953). 